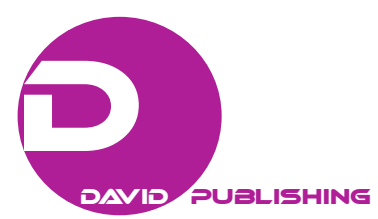

\title{
The Advent of the (Ro)bot and Their Incipient Spread in the Web
}

\author{
Massimiliano Minaudo \\ University of Palermo, Palermo, Italy
}

\begin{abstract}
Today we are practically witnessing and experiencing a real bot invasion. It is therefore permissible to ask oneself what a bot is (and it is certainly not "science fiction"). The truth is this: they are programs that respond automatically to requests/queries from human users. But where can we find them? In many fields or sectors but especially in instant messaging, for example: Whatsapp, Telegram, Facebook, or Messenger. It seems that we are talking about something complicated or very difficult, however, even we users can create a bot that can help us or others to simulate conversations or actions of the human being in order to make it easier an action or task that may be repetitive. Now it has become, in a short time, a fashion that is spreading in many sectors for its versatility. The reality is that there are many types of bots. Depending on the situation and our goal, we will choose one or the other. But let's start from the beginning and before thinking about how to create a bot, let's see what a bot is exactly.
\end{abstract}

Keywords: bot, artificial intelligence (AI), human-computer interaction (HCI)

\section{Introduction and Some Issues}

Would turn out enough onerous for any subject. Between most diffuse it is right to remember the bot planned for Internet, that is, how's the web crawler (that we can approximately indicate as “climber of the net”), nicknamed also "spider".

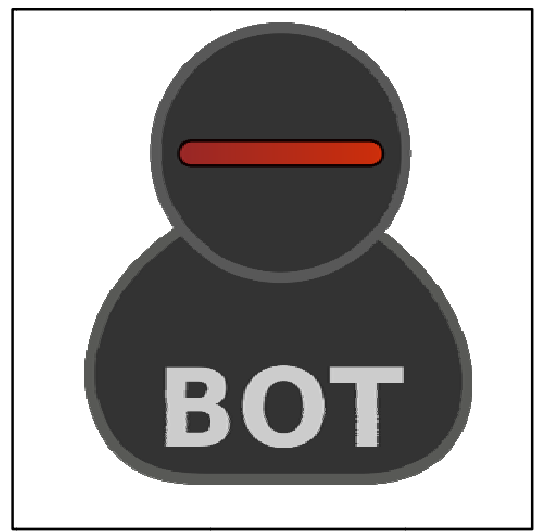

Figure 1. Bot. Source: https://it.wikipedia.org/wiki/File:Gnome-stock_person_bot.svg.

Massimiliano Minaudo, Dr., Department of Economics, Business and Statistics, University of Palermo, Palermo, Italy.

Correspondence concerning this article should be addressed to Massimiliano Minaudo, Via Fiduccia 7/A, Villabate (PA) 90039, Italy. 
The main employment of these programs is represented by the crosswalk of pages and the pursuit of hyperlinks (link) that they root out in a text in order then to pass from a page to the other, contextually, can collect information approximately the content of then was about to index them in opportune way in the main database of the search engine used from the viewer.

To better understand what we are analyzing, the author can take the example with Google Search that since 1998 has recorded one of the most famous records of indexed pages and in 2010, with the introduction of the function called "Instant Previews", which is the visualization preview of the result on the search page, has allowed Google to surpass Microsoft in the capital value on the stock exchange.

A lot interesting and less important is not the channels IRC (Internet Relay Chat) in which the bot they execute several tasks, between which: the Spamming (than addresses the customers who have made their come in a chat) and the offer of sharing (that they are inglobati in the system of the chats as soon assaid) or to succeed in "to even carry out theactive role of butlers" regarding the virtualrooms more complex.

Amazing it is the advent of the bot like "personages" you mail under the control of the computer in the games on line, better anchor, if we consider their action, it can be stated that effectively they succeed to take the place of a human player; the most common use that was discovered was to use a casual opponent, a subject who performs an exercise, a workout or simply to practice a particular activity, a training or in order to only make practice on a determined activity. Here, we assist to AI (artificial intelligence) use dedicated to the control, partial or total, of the model of same player.

There are games that, sometimes, are used through integration of "aimbot" (a name aimbot drift from the union of the two English words to aim and bot- "robot") already head for being put on of the disabled people in order to supply they a discreet level of support or aid, as in the hypothesis of the sight to take and nothing it excludes that it can be extensive also in the web in order to center the elements of a page or to focus the search on a determined point to select.

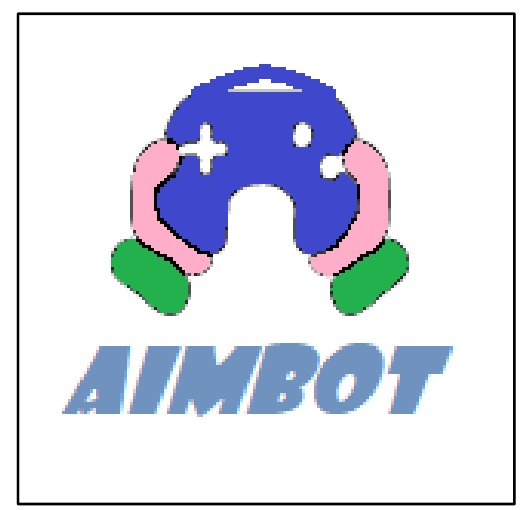

Figure 2. Aimbot example.

Clearly, it must lend much attention because online, own between the games multiplayer, the bot can even try than to take the place of a subject when it is not in its computer science emplacement and to give therefore advantage to the common players. Between the games that allow said how much is useful to remember: TimeSplitters, Half-Life 2, Unreal Tournament 2004, Rainbow Six, Call of Duty, Battlefield Play4Free, Counter-Strike.

It is necessary to specify just that there are two types of aimbot: 
- bot to color scanning;

- bot based on the device driver video.

The scanning one is effectively less used because they are calibrated on the color and when they characterize it control a pixel to the time, more over, is little effective and demands many resources even if they remain impossible to discover.

The second type is the most used and known because it intercepts the traffic between the video card and the game, moreover, works before rendering and visualization in 3d forms, exactly, the bot calculates the correct coordinates ("X" and "Y") on which then set the mouse and to do this it is based on the three-dimensional position of the mesh on the stage.

\section{Metodology}

Observation of the phenomenon, survey of the population (survey), single case study, research, meta-analysis.

\section{Research Question and Findings}

This analysis confirmed that here are the reasons for which it can be considered more efficient even if from the programs anti-cheats (created and structured even recognizable in order to avoid, for example, than the players of some games on line they can obtain undue advantages through the use of some adulterated instruments of third parts disowned to the ordinaries and unaware of sailors but by now unmasked from the experts and named "software hooks").

For the type plans wiki, we can notice a function detail, even if it is not the only one, than substantiates in the tasks of reordering automatic rifle of the pages web, for example: the compilation of the connections, the correction of eventual new addressings, the creation of special pages of synthesis or other anchor.

In this overview on bots we can not neglect the IRC (Internet Relay Chat) range which includes, not only, the execution of all the scripts, but also programs for connecting to a server (as happens when a client activates to provide access even if it differs for automatically started functions); users of the channel (public or private) can, in this case, meet and even enter on it to exchange information or communicate with each other.

The developers of an IRC bot can use the script that is already in the client or specific framework to be connected to the servers with a suitable programming language and the development of bots available and maybe readjusted is not excluded, in general, the most common use is to create a detached and functioning "guest” program within the channel (better known as “takeover").

Today, we have reached a good level of implementation regarding the past, when the "elementary ones" bot were managed commonly for the games, the current panorama leads the viewer to the supply of special services, you see the access lists for example and the database, however, many networks of it forbid the employment in order to ask for the danger that could be hidden behind a screen name that, increasing the space of the database would have to be then connected and synchronized with the servers of the net, therefore, to allow the access with the web from a bot it could make to increase the traffic and to contribute to the increase of the costs without limits or brakes (problematic not negligible but imputable to the IRC).

In the event that an IRC bot has been configured to be or represent, for example, a simple channel operator then we would see new and further problems arise (assuming in this specific case the user with certain privileges), as well as happens when they are actually installed (and not under hypothesis) on some servers with high "uptime” times (see Unix-like systems) because they are incentivized by fast and fairly tight connections, 
however, less suitable or even improper are from consider those "dial-up" (in fact, require a constantly connected bot) even if to avoid this there are now "shell providers" with accounts placed in use for the proper functioning of the bots thanks to the installation on a server thanks to the installation on server.

In general terms, on the net bot the IRC serves when the recording of the channel is not necessary (as ChanServ, EFnet or IRCnet) or when the recording is wanted to be prevented on channels that do not answer to requirement or characteristics demanded (like Undernet or Quakenet), there are therefore many types of bots and that is why we will talk about it further for completeness of information.

Given that with "bot" we refer to a software programmed to have a rather automatic operation on the network, we can confirm that today the application continues to evolve and move forward according to a process that does not leave without surprises, instant messaging now incorporates the strategy reputed as winning and compelling (since it overcomes the monotony of repetitions of actions as previously mentioned).

The best use of the bot is that of using the web pages with real-time assistance to surfers who immediately receive help, for example: in the finalization of purchases or to solve problems/difficulties that customers might have. If we wanted to create this bot or chatbot (here we talk about Artificial Intelligence), there would be a big problem that would consist in the continuous maintenance caused by the errors. The more difficulties they present and the more specialized it must be the developer and the time needed to implement the program and its execution. Companies sell their products on the Internet (eCommerce) or social networks use it continuously. Facebook itself sells products through Messenger: some sales can be made inside the social network with an instant chat as happened with the bot launched by the travel company Skyscanner (Figure 3).

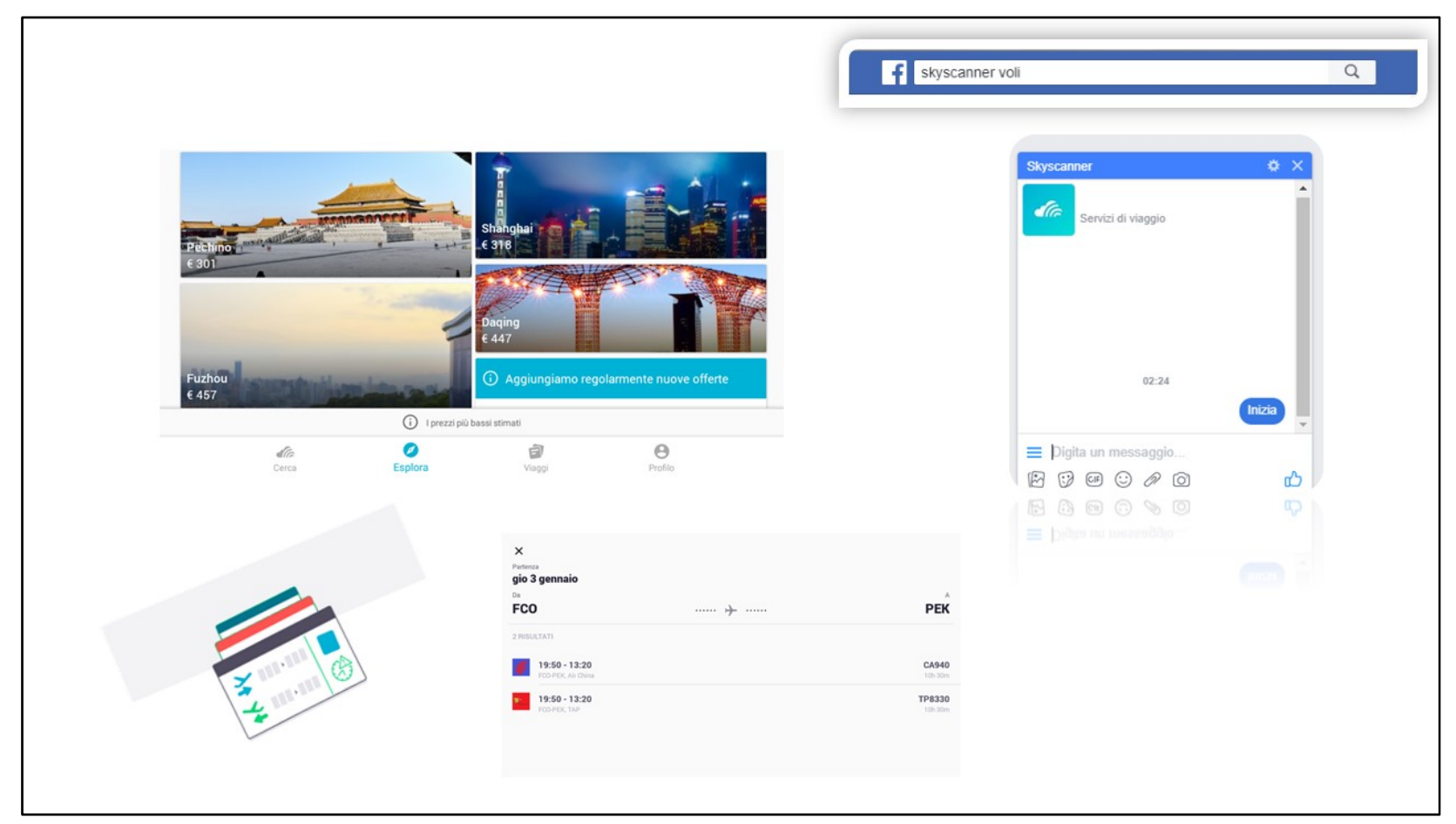

Figure 3. Skyscanner.

Simply go to the Fan Page of the brand on Facebook and send a private message. From that moment on the bot start to help you find your perfect holiday. The positive aspect of this tactic is that it allows us to give better customer support. An almost human service also attracts more customers and possible leads. 


\section{Conclusion}

In conclusion, it is not undoubtedly from pointing out like negative all this that in existence comes place with bot an IRC, indeed, on a channel could be received information of the customer upon request, be obtained statistics, more rather be accommodated sundries activity of entertainment and this by means of a precise language of scripting (Tcl/Perl) or scripts realized from the same user; there are channels that already employ bot XDCC in the distribution of rows sharing.

Keep in mind that the automation of actions in RRSS Botize or IFTTT (consists of configuring the bots to start in some situations, for example: if you tweet and write the hashtag "\#apps" to save the tweet in an Evernote list) it is one of many other possible applications.

The problems are also other and further, that is, create a bot for a company when it would cost? Is it convenient or can I create it myself? Certainly, creating it for ourselves means having good computer skills and knowing how to create an App otherwise you have to refer to experts.

A problem nestled behind all this is that of user privacy. Finally, the cost of a bot depends on our economic availability (money that we are willing to spend) and resources in our possession or even worse that we should also go to buy but these are problems related only to the end user.

Swimming under the influence of the current of bots could represent, in some ways, innovate or renew a company, make bets on the future and therefore develop better and with a growing trend the image and brand, in short, an index of modernity.

A digital marketing strategy can only be approved by the company but there may not be such a symmetrical satisfaction relationship from the client's side.

\section{References}

Al Awadi, A. H. R., \& Belaton, B. (2015). Multi-phase IRC botnet and botnet behavior detection model. DOI:10.5120/11164-6289

Bearman, A.. Russakovsky, O., Ferrari, V., \& Li, F. F. (2015). What's the point: Semantic segmentation with point supervision. Computer Vision and Pattern Recognition.

Bhute, A. N., \& Meshram, B. B. (2013). IntelligentWeb agent for search engines. International Conference on Trends and Advances in Computation and Engineering TRACE.

Conti, M., Guarisco, C., \& Spolaor, R. (2015). CAPTCHaStar! A novel CAPTCHA based on interactive shape discovery. Human-Computer Interaction.

Elyashar, A. (2016). The security of organizations and individuals in online social networks. Thesis submitted in Partial Fulfillment of the Requirements for the M.Sc. degree.

Ferrara, E., Varol, O., Davis, C., Menczer, F., \& Flammin, A. (2014). The rise of social bots. DOI:10.1145/2818717

Herath, S., Harandi, M., \& Porikli, F. (2016). Going deeper into action recognition: A survey. Computer Vision and Pattern Recognition.

Hernandez-Orallo, J. (2014). AI evaluation: Past, present and future. Journal of Artificial Intelligence Review. DOI:10.1007/s10462-016-9505-7

Josi, A., Abdillah, L. A., \& Suryayusra. (2014). Penerapan teknik webscraping pada mesin pencari artikel ilmiah. Jurnal Sistem Informasi (SISFO), 5.

Mittal, S., \& Kumaraguru, P. (2014). Broker bots: Analyzing automated activity during high impact events on Twitter. Social and Information Networks.

Oentaryo, R. J., Murdopo, A., Prasetyo, P. K., \& Lim, E. P. (2016). On profiling bots in social media. DOI:10.1007/978-3-319-47880-7_6

Pierre, J. M. (2001). On the automated classification of web sites. Link"oping Electronic Articles in Computer and Information Science, 6, 1-12. 
Subrahmanian, V. S., Azaria, A., Durst, S., Kagan, V., Galstyan, A., Lerman, K., ... \& Hwang, T. (2016). The DARPA Twitter bot challenge. IEEE Computer Magazine.

S. Haustein, T. D. Bowman, K. Holmberg, Tsou, A., Sugimoto, C. R., \& Larivière, V. (2014). Tweets as impact indicators: Examining the implications of automated bot accounts on Twitter. Social and Information Networks.

Sinha, T., \& Rajasingh, I. (2014). Towards investigating substructures and role recognition in goal oriented online communities. 4th IEEE International Advance Computing Conference (IACC), India.

Sanatinia, A., \& Noubir, G. (2015). OnionBots: Subverting privacy infrastructure for cyber attacks. Cryptography and Security.

Wu, Q., Teney, D., Wang, P., Shen, C. H., Dick, A., \& van den Hengel, A. (2016). Visual question answering: A survey of methods and datasets. Computer Vision and Pattern Recognition.

https://www.researchgate.net/publication/283854862_Espionage_A_voice_guided_surveillance_robot_with_DTMF_control_and_ web_based_control

https://www.researchgate.net/publication/301981878_Lino_An_Intelligent_System_for_Detecting_Malicious_Web-Robots

https://www.researchgate.net/publication/282644126_The_importance_of_data_mining_technologies_and_the_role_of_intelligent _agents_in_cybercrime

https://www.researchgate.net/publication/236108776_A_dynamic_URL_assignment_method_for_parallel_web_crawler 